\section{In Memoriam: Arti Hurria, MD}

\author{
June M. McKoy, MD, MPH, JD, MBA
}

\section{Unexpected Passing}

On October 11, 2018, at 2:54 PM, I sent an email to Arti Hurria, MD, indicating my desire to start writing papers relevant to the $\mathrm{R} 25$ grant for which I had been an annual presenter for the past 3 years, and that she had received to increase oncology nurses' knowledge of caring for older adults with cancer. At 4:19 PM, I received the following response, "That is wonderful, June! There is so much to write about! Is there a topic that you would like to take the lead on?" That was so like Dr. Hurria: to be inclusive, enthusiastic, and generous. And that was the last correspondence we had.

On November 7, 2018, Dr. Arti Hurria, George Tsai Family Chair in Geriatric Oncology, Director of the Center for Cancer and Aging at City of Hope, cofounder of the Cancer and Aging Research Group (CARG), and beloved leader in and champion of geriatric oncology, died in a traffic accident in Los Angeles, California. Dr. Hurria was only 48 years of age and in the prime of her life.

\section{Life's Work}

Dr. Hurria dedicated her life to the elderly in her chosen field of geriatric oncology. Outside of her family, the care and well-being of the older adult was her singular focus. In addition to improving clinical treatment approaches to the aging population, she unapologetically advocated for their inclusion in clinical trials. Her collaborative research and publications on oncology issues in the aging population established her reputation worldwide as a leader in the area of geriatric oncology. In 2006, she cofounded the first research group (CARG) dedicated and devoted to investigations in cancer in older individuals. Furthermore, she increased integration of the comprehensive geriatric assessment into every aspect of care of the older patient with cancer, including as a predictor of chemotoxicity in older individuals. This bold and precocious approach to treatment of older individuals with cancer was embraced over time and has become the accepted cancer treatment approach for older adults. As chair of the NCCN Older Adult Oncology Panel, she strove for consensus guidelines that were steeped in evidence and appropriate to the physiologic milieu of the older adult.

\section{Rising Cancer Incidence}

Patients aged 65 years and older carry the overwhelming burden of cancer; therefore, research among this population is critically important. Indeed, adults aged 65 years and older account for $60 \%$ of new cancer diagnoses, and $70 \%$ of cancer-related deaths occur in this population. ${ }^{1,2}$ Experts project that cancer incidence among individuals aged 65 years and older will increase $67 \%$ from 2010 to 2030.1,2 Despite these statistics, studies are often performed in younger populations and results are then extrapolated to the elderly.

The underrepresentation of the older adult in randomized clinical trials, resulting in a dearth of evidence to inform drug use, dosing, and schedule, is unacceptable. ${ }^{3}$ Hurria et $\mathrm{al}^{4}$ found that data on toxicity are limited for patients older than 75 years and further noted a paucity of data for patients older than 80 years. Furthermore, chronologic age and functional age are undoubtedly disparate in older individuals, and drug treatment decisions should be individualized. Therapeutic decisions must be based on measures of performance status and backed up by evidence.

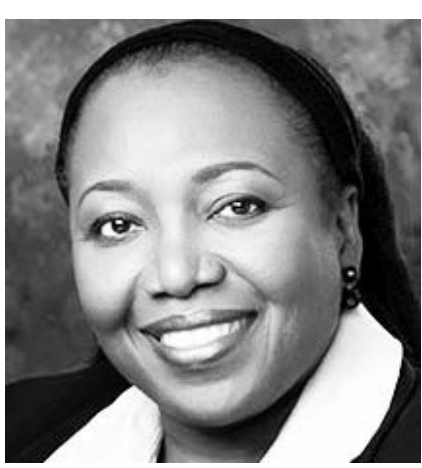

June M. McKoy, MD, MPH, JD, MBA

June M. McKoy, MD, MPH, JD, MBA, is an Associate Professor of Medicine and Preventive Medicine at Northwestern University (NU) Feinberg School of Medicine, a licensed Illinois attorney with certified expertise in Health law and Elder Law, and a NIH-funded clinical cancer/ health services researcher. A member of the Survivorship Institute of NU's Robert H. Lurie Comprehensive Cancer Center in Chicago, she co-founded the Senior Oncology Outcomes Advocacy and Research (SOAR) program that translates research on cancer health measures into advocacy based interventions to improve health-related quality of life and survivorship for older individuals. She is an advisory board member for The Cancer Legal Resource Center and serves on the NCCN Older Adult Oncology Guidelines Panel. Dr. McKoy's research is at the interface of cancer and aging, with special foci in the areas of adverse cancer drug reactions in older individuals, law and cancer health policy, and cancer survivorship. She is the Co-Lead for the Community Engagement Core of Chicago Cancer Health Equity Collaborative (ChicagoCHEC), an $\mathrm{NCl}$ funded research initiative. Furthermore, she is a member of the Cancer and Aging Research Group (CARG) housed at City of Hope Comprehensive Cancer Center, a member of the NCl's National Council of Research Advocates (NCRA), a NIH Special Emphasis Panel reviewer, and a reviewer for several scientific journals.

doi: 10.6004/jnccn.2018.0093

The ideas and viewpoints expressed in this commentary are those of the author and do not necessarily represent any policy, position, or program of NCCN. 


\section{The Hurria Legacy}

Dr. Hurria's work is documented in more than 200 papers and numerous presentations at national and international scientific meetings. It is also mirrored in numerous awards and recognitions, including an ASCO Career Development Award (2005), an ASCO Young Investigator Award (2002-2003), the BJ Kennedy Award for Scientific Excellence in Geriatric Oncology (2013; triggered by her studies to develop a treatment tolerance model for older patients), and the International Society of Geriatric Oncology (SIOG) Paul Calabrese Award (2017). Since the start of the CARG, 24 geriatric oncology protocols have been launched and have enrolled more than 3,200 older individuals. Dr. Hurria served as the founding editor-in-chief of the Journal of Geriatric Oncology and was a former president of the SIOG, solidifying her international reputation as advocate and mentor.

Dr. Hurria was also a "renaissance teacher" who recognized that the care of the older adult required a multidisciplinary approach and that the backbone of that care is nursing. With this insight, she obtained an R25 grant from the NCI to contribute to broadening the scope of geriatrics training for oncology nurses. I was honored to be invited as a teacher for that course and looked forward every year to meeting the dedicated nurses who brought their knowledge and commitment to older patients with cancer.

\section{Not the "Final Chapter"}

On November 7, 2018, Dr. Arti Hurria closed the "final chapter" in her life. However, a new chapter in her work begins: colleagues and mentees who were inspired by her mission have convened under the name "Arti's Light." This community of geriatricians and geriatric oncologists will not only carry on her legacy, but also will work with renewed fervor to ensure that additional policies are instituted to make clinical trials that include older adults the standard, rather than an anomaly.

Dr. Hurria was a disrupter in the most positive sense. She fought against the status quo that seemed comfortable in treating older adults with protocols borrowed from younger patients. In all her activities, including her tenure on the ASCO board and her publications, she called for fundamental changes to the way we treat older patients with cancer. Geriatric Oncology: Treatment, Assessment and Management, coedited by Dr. Hurria and published by Springer in 2009, remains an important reference book for medical oncologists and other healthcare providers who are caring for older adults with cancer; each chapter underscores the specific challenges of the aging patient. Indeed, the Hurria approach to the care of older adults with cancer has become the standard bearer. Every older adult cancer survivor who benefits from treatment researched and developed for them will be a living testimony to Dr. Hurria's work and the greatest part of her legacy.

Dr. Arti Hurria will be remembered with admiration and respect. Her dedication to aging research, her generosity to those she mentored, and her singular dedication to her patients were exemplary. Her light and her legacy will continue to spur generations of young oncologists to champion the cause of older adults with cancer. There is much more work to be done and we are committed to doing it. Rest in peace, my friend.

\section{References}

1. Smith BD, Smith GL, Hurria A, et al. Future of cancer incidence in the United States: burdens upon an aging, changing nation. J Clin Oncol 2009;27:2758-2765.

2. McKoy JM, Samaras AT, Bennett CL. Providing cancer care to a graying and diverse cancer population in the 21st century: are we prepared? J Clin Oncol 2009;27:2745-2746.

3. Le Saux O, Falandry C, Gan HK, et al. Inclusion of elderly patients in oncology clinical trials. Ann Oncol 2016;27:1799-1804.

4. Hurria A, Togawa K, Mohile SG, et al. Predicting chemotherapy toxicity in older adults with cancer: a prospective multicenter study. J Clin Oncol 2011;29:3457-3465. 